\title{
The effect of cytokinins on in vitro shoot length and multiplication of Hymenocallis littoralis
}

\author{
Chai Kian Yew, Bhavani Balakrishnan, Jeevandran Sundasekaran and \\ Sreeramanan Subramaniam*
}

School of Biological Sciences, University Sains Malaysia, Minden Heights, 11800 Penang, Malaysia.

Accepted 8 October, 2010

\begin{abstract}
This study was performed to determine the effects of different cytokinins at various concentrations on the in vitro shoot formation of Hymenocallis littoralis. The bulb scales of $\boldsymbol{H}$. littoralis were used as explants to establish the cultures. The explants were grown on semi-solid Murashige and Skoog (MS) medium supplemented with $2 \mathrm{iP}$ (2-isopentenyladenine), TDZ (thidiazuron) and Zeatin respectively at six different concentrations $(2.25,4.50,9.00,13.50,18.00$, and $22.50 \mu \mathrm{M})$. Two subcultures were performed at 30 days interval after the initial in vitro culture establishments. It was found that $2 \mathrm{iP}$ and concentration $13.50 \mu \mathrm{M}$ respectively was the best for shoot elongation which was measured in length (cm). However, the three cytokinins and the six tested concentrations were recorded to have no significant difference in terms of shoot multiplication. Highest total chlorophyll content was observed in shoots grown on semi-solid MS medium supplemented with 2.25 $\mu \mathrm{M}$ of Zeatin.
\end{abstract}

Key words: Hymenocallis littoralis, in vitro, cytokinins.

\section{INTRODUCTION}

Hymenocallis littoralis (Jacq.) Salisb. is a bulbous, herbaceous plant from the family Amaryllidaceae. It is also known as Hymenocallis panamensis Lindl., Pancratium americanum Mill., Pancratium littorale Jacq. (loset et al., 2001; Ocampo and Balick, 2009). The plant is distributed by the sea and in swamps in tropical, subtropical, and temperate regions throughout the world ( $\mathrm{Ji}$ and Meerow, 1985).

Other than its application as an ornamental plant, $\mathrm{H}$. littoralis was discovered to exhibit various medicinal properties (Backhaus et al., 1992). Indeed, many plants from the same family (Amaryllidaceae) as $\mathrm{H}$. littoralis had served as cures of various illnesses for decades. In particular, the bulbs of Hymenocallis Americana were made into poultice for varicose veins, sores and swellings. Hymenocallis amancaes is commonly employed as an ornamental plant and used in cosmetic preparations.

Applying the mixture of oil and crushed bulbs onto the

${ }^{\overline{ }}$ Corresponding author. E-mail: sreeramanan@gmail.com. Tel: 60-16-4141109. Fax: 60-4-6565125. face has been found to treat freckles and blemishes (Ocampo and Balick, 2009). Hence, it is obvious that the various medicinal applications of plants in the genus Hymenocallis come primarily from their bulbs. In fact, almost all of the studies on $\mathrm{H}$. littoralis were done on its seemingly most valuable part, the bulb (loset et al., 2001). Throughout the history of $H$. littoralis, several alkaloids have been discovered from its bulb. The first alkaloid, lycorine was proven to have antineoplastic, cytotoxic and antiviral properties (loset et al., 2001). Another compound, pancratistatin has been proven to be effective against U.S. National Cancer Institute's panel of 60 human cancer cell lines demonstrating greatest effectiveness against melanoma, brain, colon, lung and renal cancers (Backhaus et al., 1992; Pettit et al., 1993). Littoraline alkaloid was then discovered with inhibitory activity on HIV reverse transcriptase (Lin et al., 1995). As the compounds isolated from $H$. littoralis have been proven to demonstrate various medicinal properties (Lin et al., 1995; Idso et al., 2000), it acts as the driving force behind the current study to micropropagate the plant using plant tissue culture techniques.

Micropropagation is the in vitro asexual multiplication of 
genetically identical individuals using plant tissue culture technology. Cytokinin is one of the plant hormones crucial for plant growth and development and is known to promote cell division. Various types of cytokinins can also stimulate lateral bud growth and thus causing multiple shoot formation by breaking shoot apical dominance (Trigiano and Gray, 2005). Cytokinins are categorically divided into naturally-occurring and synthetic. Out of the three cytokinins used in the present study, 2iP (2isopentenyladenine) and Zeatin are naturally-occurring while TDZ (thidiazuron) is synthetic and highly active (Schmulling, 2004). The specific objectives of this study are (i) to determine the growth of $H$. littoralis in semi-solid MS medium supplemented with three types of cytokinins namely 2iP (2-isopentenyladenine), TDZ (thidiazuron) and Zeatin at six different concentrations $(2.25,4.50$, $9.00,13.50,18.00$, and $22.50 \mu \mathrm{M}$ ); (ii) to select the most effective cytokinin and its concentration for in vitro micropropagation of $H$. littoralis in terms of shoot growth (length) and shoot multiplication, and (iii) to select the best cytokinin and its concentration, in terms of highest total chlorophyll content, for the in vitro micropropagation of $H$. littoralis.

\section{MATERIALS AND METHODS}

\section{Bulb preparation}

The explant used in this study was the bulb of $H$. littoralis. The plants were ordered from a nursery. In order to obtain the bulb, the outer black polyethylene cover and the soil covering the bulb were removed. Then, without damaging the bulb structure, the leaves and roots of the bulb were carefully cut off.

\section{Bulb surface sterilization and dissection}

The brown coating of the bulb was peeled off, revealing a whole "clean" white bulb. It was then placed in a beaker and washed with tap water added with 2 to 3 drops of Teepol for an hour to thoroughly remove all of the attached dirt, debris, and soil.

The bulb was then dissected into four parts equally. Before further dissection, surface sterilization procedures were conducted on the divided tiller shoots. Firstly, they were immersed in $70 \%$ ethanol for $2 \mathrm{~min}$ and transferred to $50 \%$ sodium hypochlorite solution (Clorox 5.5\%) for $20 \mathrm{~min}$. Upon completion of the sodium hypochlorite wash, subsequent steps were carried out in aseptic conditions in the laminar flow hood. A second immersion in $70 \%$ ethanol for another $2 \mathrm{~min}$ is followed by rinsing the tissues three times in distilled water. The tissues were blot dried on a filter paper prior transferring under aseptic condition.

\section{Culture establishment}

To initiate a culture, the basal vascular portion of explant was planted into the semi-solid agar medium. At every concentration out of the six concentrations $(2.25,4.50,9.00,13.50,18.00$, and 22.50 $\mu \mathrm{M})$ for $2 \mathrm{iP}, \mathrm{TDZ}$ and Zeatin, four explants were planted on the semi-solid MS medium (Murashige and Skoog, 1962) in a single culture jar. It was then repeated for three times which made up to (4 explants $\times 4$ jars) a total of 16 replicates. All of the cultures were placed on a white wooden rack in the culture room at $25 \pm 2{ }^{\circ} \mathrm{C}$ with continuous cool white fluorescent light. The cultures were grown for 30 days which was the interval for a subculture. Two subcultures had been performed in this study.

\section{Shoot length and multiplication}

After a period of 30 days from the initial culture establishment date, all of the plantlets were taken out, one culture jar after another, and placed on a sterilized Petri dish under which a graph paper was adhered to. The morphology of all the plantlets was carefully observed and compared to each other. Then, the number of shoots and their respective lengths were measured and recorded. All of the procedures were done under strict aseptic techniques and conditions as the contamination of any culture would require it to be discarded. After the measurements were completed, all of the plantlets were transferred to a culture jar containing new semi-solid MS medium according to their respective cytokinin type and concentration. From here, the first subculture was done and the steps were repeated after another 30 days for the second subculture.

\section{Chlorophyll content}

When the second subculture was completed, the plantlets were used to determine their total chlorophyll content using Harborne method (Harborne, 1973).

\section{RESULTS AND DISCUSSION}

\section{Shoot length}

$H$. littoralis shoots start to form within one week from the day of culture establishment. The success rate of shoot formation was at an average of $93.4 \%$.

Figure 1 shows mean shoot length $(\mathrm{cm})$ against the six concentrations used for 2iP, TDZ and Zeatin after the first and the second subcultures. The cytokinin concentration of $13.50 \mu \mathrm{M}$ was found to be the best for stimulating shoot length followed by $18.00,22.50,9.00,2.25$ and $4.50 \mu \mathrm{M}$. Nevertheless, based on Dunnett's test (2-sided) of multiple mean comparisons, only 13.50 and $18.00 \mu \mathrm{M}$ were significantly different from other concentrations tested in terms of mean shoot length. While the mean shoot length increased from $2.25 \mu \mathrm{M}$ up to $13.50 \mu \mathrm{M}$ (the highest), it started to decrease when the cytokinin concentration was further increased. This phenomenon is actually similar to a research on micropropagation of different banana cultivars using scalps where the shoot length was increased with higher BAP (benzyl amino purine) level until $22.2 \mu \mathrm{M}$ after which the shoot length also began to fall (Shirani et al., 2010).

Figure 2 shows mean shoot length $(\mathrm{cm})$ against the type of cytokinins used in the study; 2iP, TDZ and Zeatin. Both figures recorded $2 \mathrm{iP}$ to produce the highest mean shoot length followed by TDZ and Zeatin. After the first subculture, both TDZ and Zeatin were significantly different from 2iP. Meanwhile after the second subculture, only Zeatin was significantly different from $2 \mathrm{iP}$. This observation is similar to a research on the regeneration of 
A

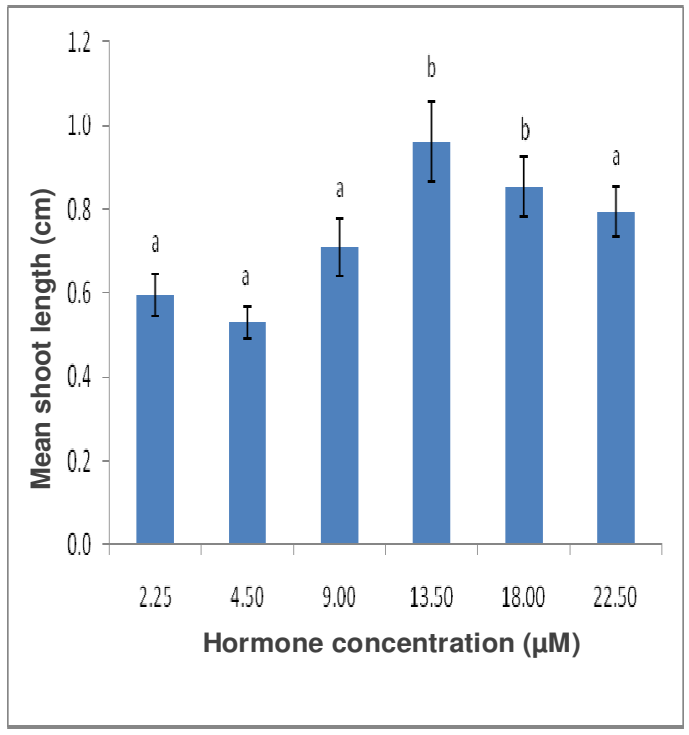

B

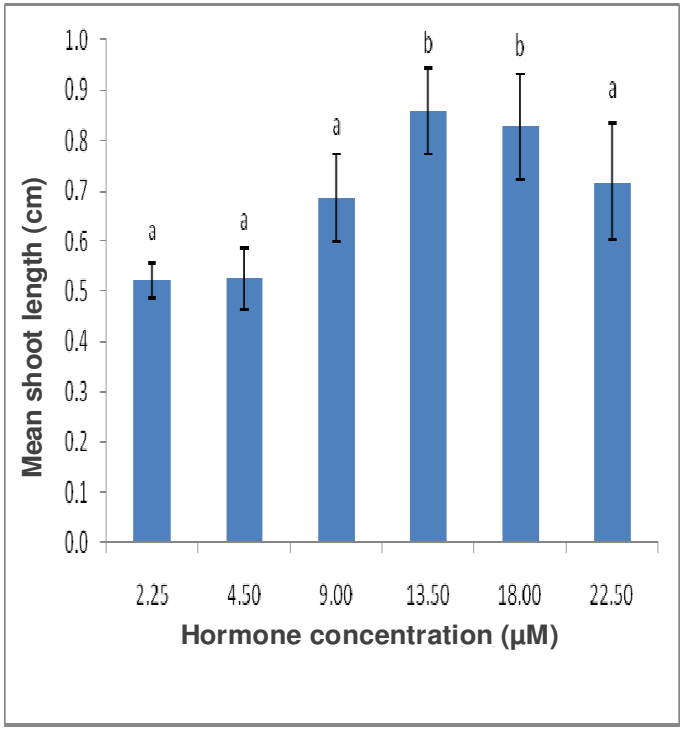

Figure 1. Mean shoot length produced by $H$. littoralis under different concentrations of various cytokinins (2iP, TDZ and Zeatin) on semi-solid MS medium. (A) After 30 days of cultivation. (B) After 60 days of cultivation. Mean with the same alphabet on top of every bar is not significantly different [Dunnett's test (2sided), $\alpha=0.05$.

A

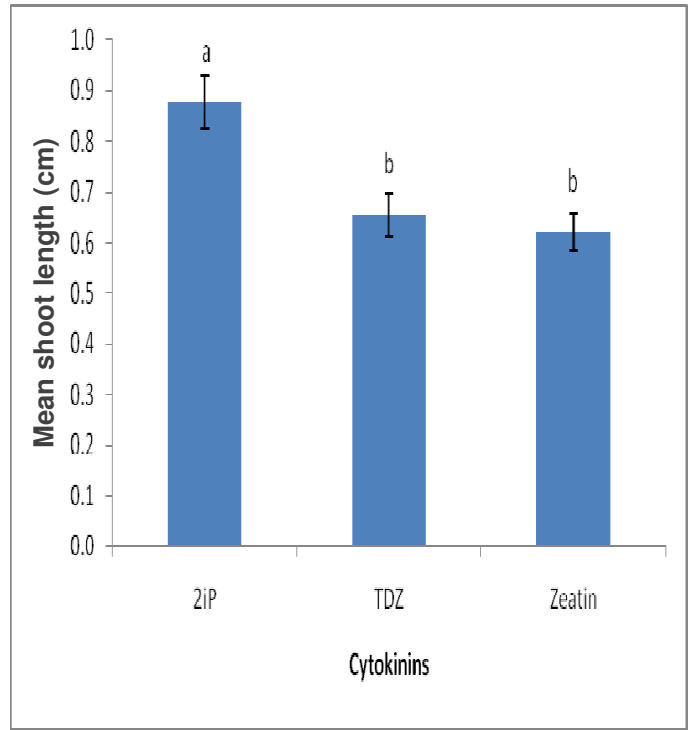

B

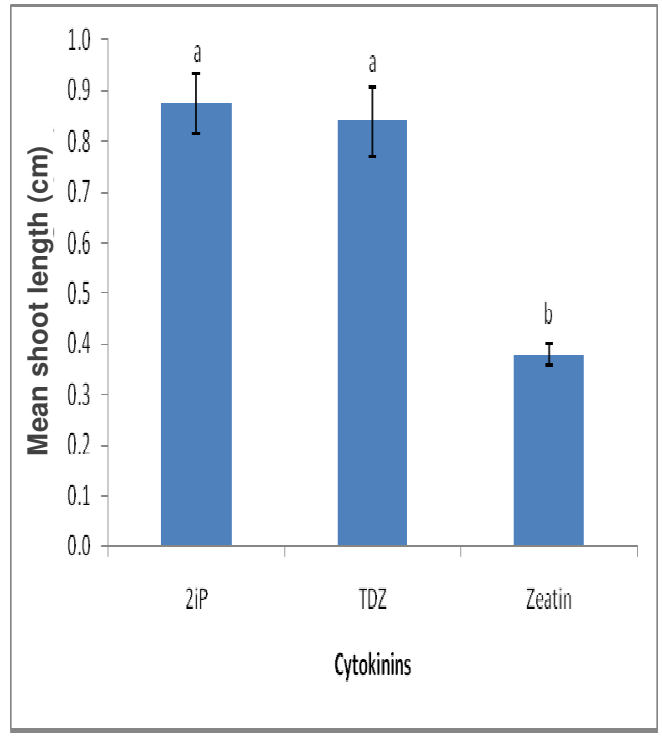

Figure 2. Mean shoot length produced by $H$. littoralis under different cytokinin treatments of various concentrations $(2.25,4.50,9.00,13.50,18.00$, and $22.50 \mu \mathrm{M})$ on semi-solid MS medium. (A) After 30 days of cultivation. (B) After 60 days of cultivation. Mean with the same alphabet on top of every bar is not significantly different [Dunnett's test (2-sided), $\alpha=0.05$ ].

sugarcane using various cytokinins (2iP, Benzyl adenine $[B A]$, Kinetin, TDZ, and Zeatin) where 2iP had been reported to be the most effective for shoot elongation (Chengalrayan and Gallo-Meagher, 2001). The low mean shoot length of TDZ in the first subculture was because TDZ had been found to reduce shoot elongation (Bates et al., 1992; Murthy et al., 1998). However, if the culturing period was increased, the shoots would eventually 
A

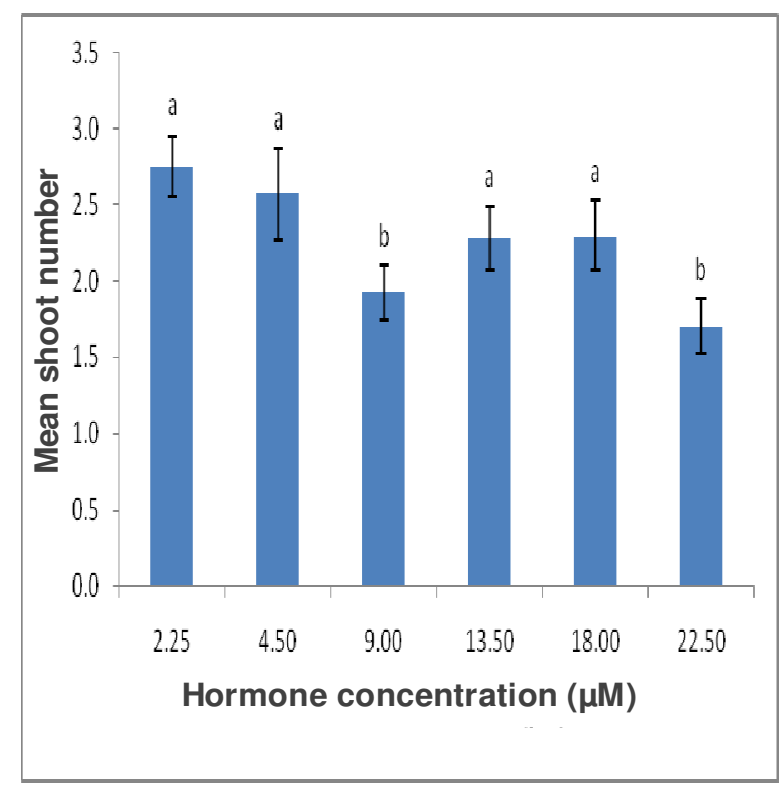

B

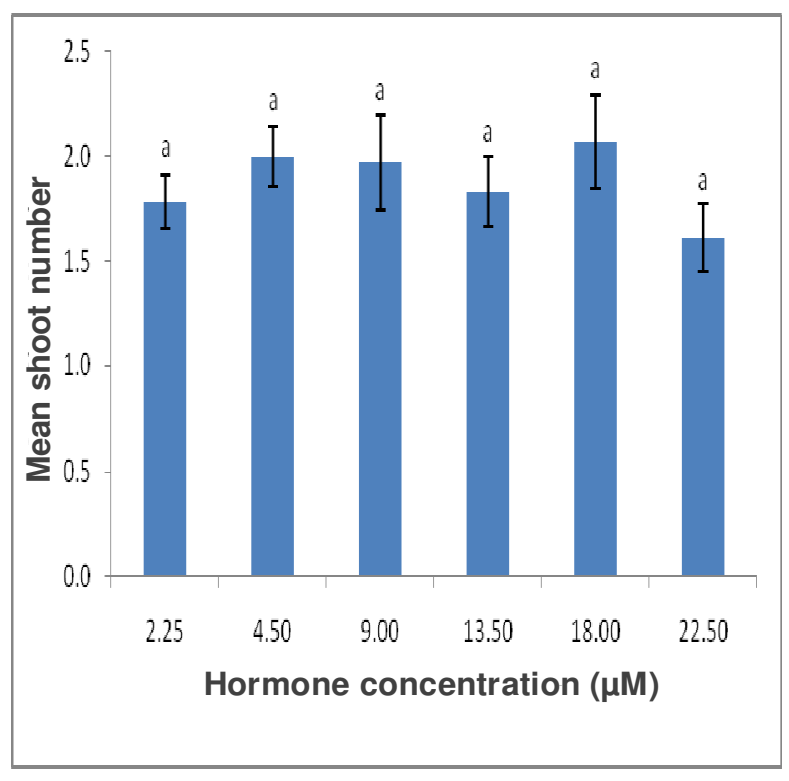

Figure 3. Mean shoot number produced by $H$. littoralis under different concentrations of various cytokinins (2iP, TDZ and Zeatin) on semi-solid MS medium. (A) After 30 days of cultivation. (B) After 60 days of cultivation. Mean with the same alphabet on top of every bar is not significantly different [Dunnett's test (2-sided), $\alpha=0.05$ ].

elongate well. Indeed, various studies showed that TDZ was effective and more active than benzyl adenine (BA) and Zeatin especially in the micropropagation of woody plants (Lu, 1993).

\section{Shoot multiplication}

The mean shoot number produced using $2.25 \mu \mathrm{M}$ was the highest after 30 days of cultivation followed by 4.50 $\mu \mathrm{M}, 13.50$ or $18.00 \mu \mathrm{M}, 9.00 \mu \mathrm{M}$, and $22.50 \mu \mathrm{M}$ (Figure $3 A)$. Dunnett's test (2-sided) of multiple mean comparisons indicated that 9.00 and $22.50 \mu \mathrm{M}$ were significantly different from $2.25 \mu \mathrm{M}$. Since explants usually require a period of adaptation to in vitro environment, this may be the cause of significantly lower mean shoot number at concentrations 9.00 and $22.50 \mu \mathrm{M}$ from $2.25 \mu \mathrm{M}$ after the first subculture (Carelli and Echeverrigaray, 2002). However, after the second subculture (Figure 3B), statistical analysis revealed that there was no significant difference between the six concentrations tested. This may be because the cytokinin concentrations used were not high enough to effectively stimulate rapid shoot multiplication. Previous studies involving other plants noted that relatively high concentrations of cytokinins should be present to observe high multiplication rates. In the case of Gerbera cultivars multiplication, it was reported that the supplementation of $10 \mathrm{mg} / \mathrm{L}(44.40 \mu \mathrm{M})$ benzyl adenine (BA), twice the maximum cytokinin concentration used in this study, increased adventitious bud formations in all cultivars during the initial and the multiplication stages (Rael and Tõnis, 2001).

The relationship between mean shoot number and the cytokinins used is shown in Figure 4. There was no significant difference in terms of mean shoot number among the three cytokinins. Further statistical analysis indicated that the effect of the three cytokinins on mean shoot number was not significantly different between the first and the second subculture periods. Cytokinin alone may not be able to significantly induce multiple shoot formation in $\mathrm{H}$. littoralis. Some studies demonstrated that the addition of auxin in synergy with cytokinin promotes better shoot proliferation (Chengalrayan and GalloMeagher, 2001). Another suggestion would be to supply combinations of cytokinins. In a study concerning Bauhinia vahlii, a leguminous plant, the combination of TDZ and Kinetin recorded significant increase in shoot numbers lasting for as long as 4 subculture periods (Bhatt and Dhar, 2000).

\section{Chlorophyll content}

For TDZ and Zeatin, the overall pattern of total chlorophyll content was seen to be descending from the lowest concentration $(2.25 \mu \mathrm{M})$ to the highest concentration $(22.50 \mu \mathrm{M})$ (Figure 5). This was translated as lower cytokinin concentration stimulating higher chlorophyll content and vice versa. Although these two cytokinins demonstrated similar descending pattern, 
A

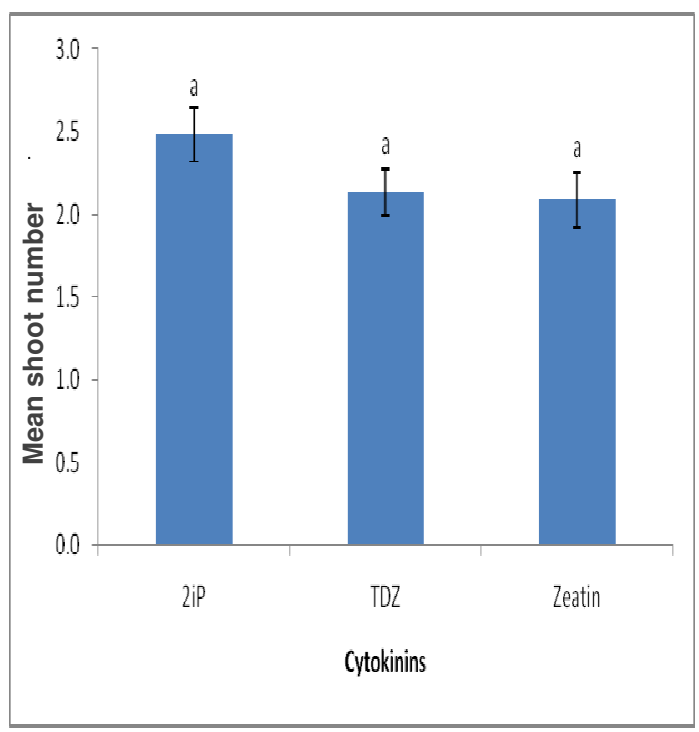

B

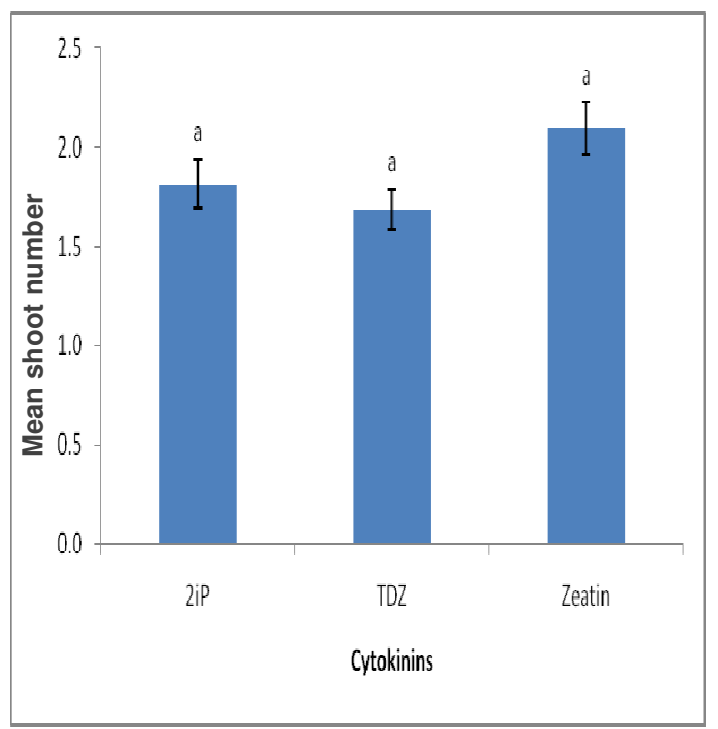

Figure 4. Mean shoot number produced by $H$. littoralis under different cytokinin treatments of various concentrations $(2.25,4.50,9.00,13.50,18.00$, and $22.50 \mu \mathrm{M})$ on semi-solid MS medium. (A) After 30 days of cultivation. (B) After 60 days of cultivation. Mean with the same alphabet on top of every bar is not significantly different [Dunnett's test (2-sided), $\alpha=0.05$.

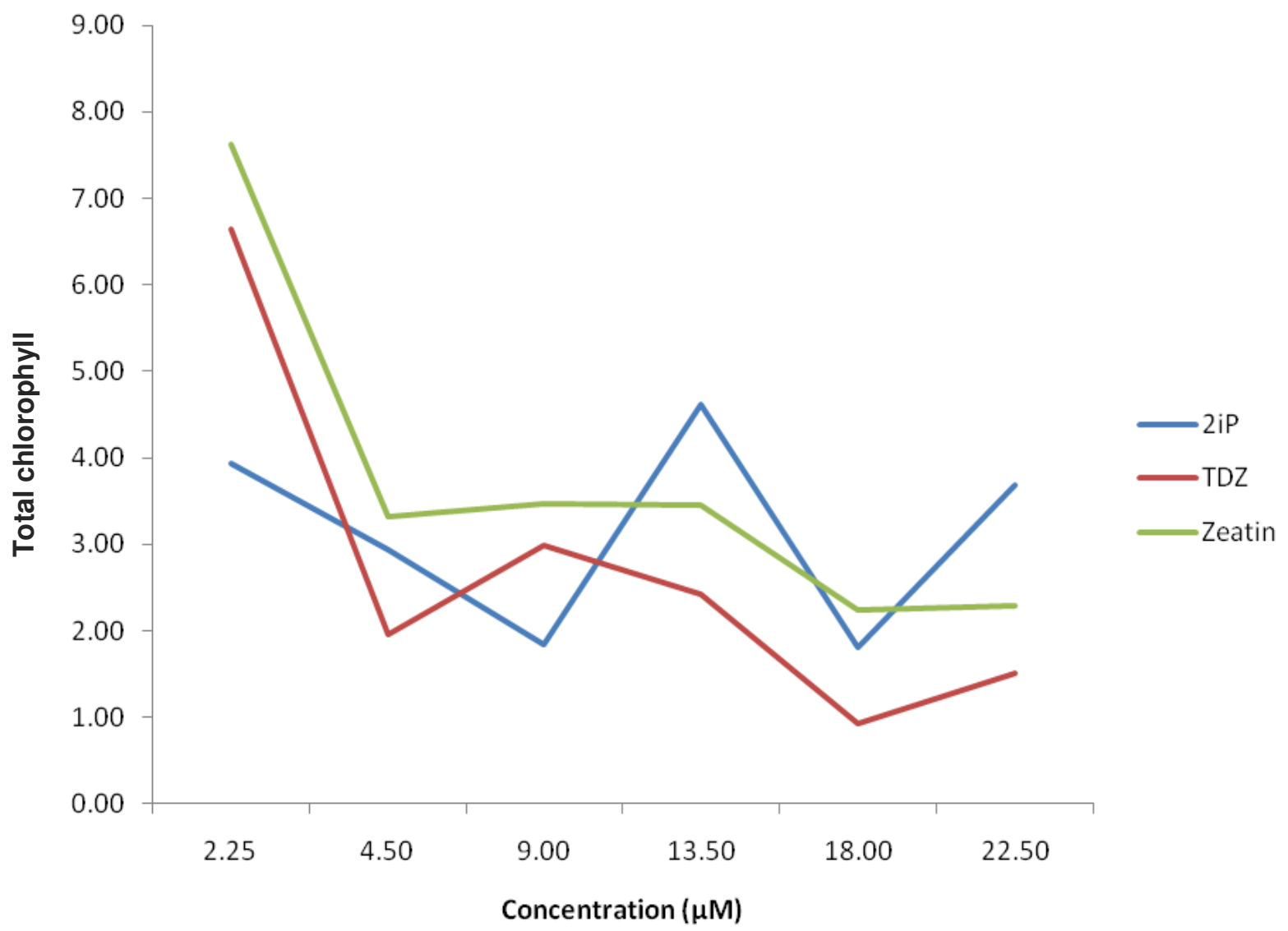

Figure 5. Total chlorophyll content of $H$. littoralis under different concentrations $(2.25,4.50,9.00,13.50,18.00$, and $22.50 \mu \mathrm{M})$ for $2 \mathrm{iP}$, TDZ and Zeatin on semi-solid MS medium after 60 days of cultivation. 
Zeatin deposited higher total chlorophyll contents than TDZ in all of the six tested concentrations. This situation occurred because Zeatin was able to induce chloroplast differentiation but TDZ was known to inhibit the process (Oliveiraa et al., 2008). 2iP registered a fluctuating pattern between the total chlorophyll content of 2.00 and 5.00 across the six tested concentrations. The fluctuation can be explained by comparing the Figure 5 . Before the first subculture, no plantlet in 2iP, TDZ or Zeatin demonstrated root growth. However, 2iP stimulated root growth especially at lower concentrations, prior to the second subculture. One of the characteristics of in vitro plantlets is the tendency to assume heterotrophic rather than autotrophic mode of nutrition as the nutrients are abundantly supplied in the medium. Hence, instead of depositing chlorophyll to synthesize food, the regenerated roots in $2 \mathrm{iP}$ plantlets directly absorbed nutrients from the medium resulting in unpredictable total chlorophyll contents across different concentrations (Hazarika, 2006). Nevertheless, explants treated with $13.50 \mu \mathrm{M}$ of $2 \mathrm{iP}$ recorded the highest total chlorophyll content.

In conclusion, the effect of cytokinins 2iP, TDZ and Zeatin at various concentrations on the micropropagation of Hymenocallis littoralis using bulb scale discovered that 2iP and $13.50 \mu \mathrm{M}$ respectively to be the best for shoot elongation. The types of cytokinins and their concentrations did not demonstrate any significant difference on the shoot multiplication of $H$. littoralis. The highest chlorophyll content was found to be in explants treated with $2.25 \mu \mathrm{M}$ of Zeatin due to its ability to stimulate chloroplast differentiation. When the chlorophyll content analysis was considered specifically for 2iP, $13.50 \mu \mathrm{M}$ registered the highest total chlorophyll content out of the total six concentrations. This finding indeed strengthened the conclusion that $2 \mathrm{iP}$ and $13.50 \mu \mathrm{M}$ were the best for shoot elongation as shoots are one of the main depositories of chlorophyll for photosynthesis.

\section{REFERENCES}

Backhaus RA, Pettit III GR, Huang DS, Pettit GR, Groszek G, Odgers JC, Ho J, Meerow A (1992). Biosynthesis of the antineoplastic pancratistatin following tissue culture of Hymenocallis littoralis (Amaryllidaceae). Acta Horticult., 306: 364-366.

Bates S, Preece JE, Navarrete NE, Van Sambeek JW, Gaffney GR (1992). Thidiazuron stimulates shoot organogenesis and somatic embryogenesis in white ash (Fraxinus americana L.). Plant Cell, Tissue and Organ Cult., 31:21-29.
Bhatt I, Dhar U, (2000). Combined effect of cytokinins on multiple shoot production from cotyledonary node explants of Bauhinia vahlii. Plant Cell, Tissue and Organ Cult., 62: 79-83.

Carelli BP, Echeverrigaray S (2002). An improved system for the in vitro propagation of rose cultivars. Sci. Horticult., 92: 69-74.

Harborne JB (1973). Photochemical Methods. Chapmann and Hall Ltd., London.

Hazarika BN (2006). Morpho-physiological disorders in in vitro culture of plants. Sci. Horticult., 108: 105-120.

Idso SB, Kimball BA, Pettit III GRP, Garner LC, Pettit GR, Backhaus RA (2000). Effects of atmospheric CO2 enrichment on the growth and development of Hymenocallis littoralis (Amaryllidaceae) and the concentrations of several antineoplastic and antiviral constituents of its bulbs. Am. J. of Bot., 87: 769-773.

loset JR, Marston A, Gupta MP, Hostettmann K (2001). A methylflavan with free radical scavenging properties from Pancratium littorale. Fitoterapia, 72: 35-39.

Ji Z, Meerow AW (1985). Amaryllidaceae. Flora of China, 24: 264.

Lin LZ, Hu SF, Chai HB, Pengsuparp T, Pezzuto JM, Cordell GA, Ruangrungsi N (1995). Lycorine alkaloids from Hymenocallis littoralis. Phytochemistry, 40: 1295-1298.

Lu CY (1993). The use of thidiazuron in tissue culture. In vitro Cellular and Developmental Biology - Plant, 29: 92-96.

Murashige T, Skoog F (1962). A revised medium for rapid growth and bio assays with tobacco tissue cultures. Physiologia Plantarum, 15: 473-497.

Murthy BNS, Murch SJ, Saxena PK (1998). Thidiazuron: A potent regulator of in vitro plant morphogenesis. In vitro Cellular and Developmental Biology - Plant, 34: 267-275.

Ocampo R, Balick MJ (2009). Hymenocallis littoralis (Jacq.) Salisb., in Plants of Semillas Sagradas: An Ethnomedicinal Garden in Costa Rica. Finca Luna Nueva Extractos de Costa Rica, S.A.

Oliveira LM, Paiva R, Santanaa JRF, Alves E, Nogueira RC, Pereira FD (2008). Effect of cytokinins on in vitro development of autotrophism and acclimatization of Annona glabra L. In vitro Cellular and Developmental Biology - Plant, 44: 128-135.

Pettit GR, Pettit III GR, Backhaus RA, Boyd MR, Meerow AW (1993). Antineoplastic agents, 256. Cell growth inhibitory isocarbostyrils from Hymenocallis. Journal of Natural Products 56: 1682-1687.

Schmulling T (2004). Cytokinin, In Encyclopedia of Biological Chemistry. Academic Press/Elsevier Science.

Shirani S, Sariah M, Zakaria W, Maziah M (2010). Scalp induction rate responses to cytokinins on proliferating shoot-tips of banana cultivars (Musa spp.). Am. J. Agric. Biol. Sci., 5: 128-134.

Trigiano RN, Gray DJ (2005). Plant growth regulators in plant tissue culture and development, in Plant development and biotechnology. CRC Press.

Yadav SP, Ibaraki Y, Dutta Gupta S (2010). Estimation of the chlorophyll content of micropropagated potato plants using RGB based image analysis. Plant Cell, Tissue and Organ Cult., 100: 183188. 